\title{
Writing For Entrepreneurs: Engage Through Clarity
}

\author{
Kevin P. Taylor (Stetson University)
}

"Not academic research."

I have a confession to make. As a working entrepreneur prior to earning my doctorate, this would have been my response if asked where I turn for information on running my business. I'm sure I was not alone among my fellow entrepreneurs. So why don't more entrepreneurs leverage academic writing and research when building their businesses?

\section{What Entrepreneurs Need}

Think about an entrepreneur's needs. He or she may need more-or better-customers, lower customer acquisition costs, product validation, better negotiation outcomes. The list of needs is endless. The common denominator is immediacy.

How can you, as an academic or researcher, travel the formidable distance to meet entrepreneurs where they are, from your knowledge of theory and empirical research to their need for actionable ideas and prescriptive guidance?

Translating evidence-based knowledge into value for entrepreneurs is like translating an invention into an innovation. The most technologically impressive invention becomes an innovation only once someone figures out how to use it to generate value for someone. Consider the following suggestions for tweaking your writing so entrepreneurs want to read it and discover its value.

\section{Start Strong}

First, you must do no harm-i.e., lose readers before they've begun. A good lead, or opening paragraph, accomplishes this. Hannah Block (https://training.npr.org/2016/10/12/leads-are-hardheres-how-to-write-a-good-one/) writes that the lead should "make the reader want to stay and spend some precious time with whatever you've written." Whether you choose a just-the-facts, straight news lead, an anecdotal lead, or some other, do so to entice the reader to continue.

Talk to any journalist, though, and you'll likely hear writing good leads is hard. So, if nothing else, heed Block's advice to keep your lead simple and clear, while avoiding clichés and strained cleverness.

\section{Offer a Clear So What?}

Make it clear early why the article is relevant. Years ago I tried to improve my public speaking skills. One lesson that stuck with me from that awkward experience was to be clear why an audience member should care about what I had to say.

Jerry Weissman(https://www.amazon.com/PresentingWin-Telling-Expanded-paperback/dp/0134093283) calls this the "what's in it for you?," or WIIFY.

Tell the audience clearly why they should listen-or in your case, read. What reward is awaiting them for their effort? Consider cutting out anything that doesn't help deliver on that promise of value.

\section{Don't Dumb Down Your Message}

Academic researchers typically explore the how and why of phenomena. Entrepreneurs, though, need the what - as in, what do I do? They want prescriptions. This may seem a daunting disconnect between an academic writer and an entrepreneurial reader. But, you can overcome the disconnect. Start with changing your piece's language without watering down its message. Research findings are specific and nuanced, and the Network for Business Sustainability suggests you don't want to lose those qualities of your research when translating it for practitioners. Entrepreneurs are smart people, but they are unforgiving toward obtuse academic-babble, so follow NBS's recommendation to simplify your language:

Don't use jargon or long, complex sentences or paragraphs. Practitioners like anecdotes and stories that breath [e] life into research findings.... You have to speak differently (have a different writing voice) when writing for popular or business audiences. Academic voice will ensure no readers. Read a bit on copywriting-i.e. marketing writing. It is needed to 'hook' casual readers. 
William Zinsser(https://www.amazon.com/Writing-WellClassic-Guide-Nonfiction/dp/0060891548) (p. 15) implores to choose clarity over clutter:

Beware, then, of the long word that's no better than the short word: "assistance" (help), "numerous" (many), "facilitate" (ease), "Individual" (man or woman), "remainder" (rest), "initial" (first), "implement" (do), "sufficient" (enough), "attempt" (try), "referred to as" (called), and hundreds more. Beware of all the slippery new fad words: paradigm and parameter, prioritize and potentialize. They are all weeds that will smother what you write. Don't dialogue with someone you can talk to. Don't interface with anybody.

Beyond the words you choose, the medium also matters. Blogging trainer Jon Morrow (https://smartblogger.com/how-to-write-a-paragraph/) suggests digital writing must have a different shape and composition than print. Online readers tend toward skimming-hunting for helpful nuggets-rather than deep reading. To improve skim-ability, consider aiming for about 150 words per paragraph.

Indeed, beware monotonous paragraph after monotonous paragraph.

Sprinkle in one-sentence paragraphs to make your prose feel more dynamic. In addition, use headers and bulleted lists as signposts for the skimmers hunting through your article.

\section{End By Delivering For The Reader}

Consider how to treat those who finish your article. Reward them for sticking it out to the end by wrapping up in a "decisive and interesting fashion." (https://blogs.Ise.ac.uk/impactofsocialsciences/2016/01/ 25/how-to-write-a-blogpost-from-your-journal-article/) Good endings for entrepreneurs, of course, will offer some practical guidance-this is what entrepreneurs seek. This is what skimmers hunt for. If you don't have specific recommendations to offer, include a closing insight that puts a bow on the article. Perhaps revisit a question, character, or dilemma from earlier in the article. Then again, one of the best gifts to a busy reader may be to just end quickly (https://training.npr.org/2016/07/29/how-to-write-short/)

\section{Conclusion}

Entrepreneurship professors impact the next generation of entrepreneurs each day in their classrooms. Their research efforts rarely show commensurate impact on entrepreneurship practitioners. If we believe our insights are important and useful-and if we didn't, then we're in the wrong line of work-then each of us must close the distance to the entrepreneur through our writing style. In so doing, we have a hope of impacting the entrepreneurship community beyond classrooms and journals. 